\title{
SPATIAL THINKING AND LEARNING ASTRONOMY: THE IMPLICIT VISUAL GRAMMAR OF ASTRONOMICAL PARADIGMS
}

Lon Clay Hill, Jr.

Dept. of Physical Science, Broward Community College - Central, Fort Lauderdale, Florida 33314, U.S.A.

As several previous speakers have noted, astronomers are a rare breed. Even though we represent the oldest science, we are as rare as poets. The fact that our discipline is so distinct suggests that it should also have its peculiar pedagogical problems. I have been attempting to understand a pervasive element of all astronomical thinking - the use of spatial imagination to link celestial phenomena to terrestrial analogs. My work has two components - a theoretical explication of astronomical thinking and some practical use of these ideas in teaching situations.

If one looks at some of the fundamental "facts" discovered by astronomers, we will find that we often do not, in fact, directly verify our discoveries as scientists supposedly do. Primary astronomical "truths" such as the sphericity of the Earth, the heliocentric orbit of the Earth, the identification of the stars as distant suns, and the recognition that galaxies are enormous collections of stars, were not discovered by direct verification. Thus, we note that the Greeks discovered the Earth's sphericity nearly two millennia before its circumnavigation and that only a few thousand very bright stars in nearby galaxies have been resolved in our largest telescopes. Some of the primary objects of astronomical discourse, then, are not perceptible objects, but objects created by the spatial imagination.

Likewise, our methodologies are replete with analogies to perceptual expericnce that cannot be directly tested over the next few millennia. To cite a few, we assunne that stellar parallax is similar to stereoscopic parallax, that the periodic diminution of the light of an eclipsing binary is due to occlusion and that the retrograde motion of planets and the proper motion of stars are due to differential-motion parallax. We are convinced of the appropriateness of these methodologies precisely because we see them as straightforward extensions of procedures taken for granted in our ordinary lives.

As astronomers, of course, we are successful precisely because we do not spend time wondering about these leaps of geometric and spatial imagination. However, as teachers of astronomy, we must make the spatial content of fundamental astronomical relationships transparent to students who do not possess unusual spatial thinking abilities. For my dissertation work at the University of Iowa, I am using both traditional and novel materials. This work includes extensive use of palpable models in both lecture demonstrations and laboratory exercises, an eclipsing-binary computer exercises in which students must choose pairs of stars that generate a set of given light curves, and a number of stereolessons that use stereograms to intro- 
duce students to important 3-D relationships. The underlying assumption is that if the students have a direct image of our "object" they can employ their full intuitive powers instead of merely those formal paradigms under their conscious control. If we as teachers thus make our spatial assumptions perceptually evident, we will find that our students will more surely use the seemingly technical tools of our trade.

\section{References}

Lon Clay Hill, Jr., "Astronomical Imagination and Stereoscopic Vision: Conscious and Unconscious Processing of Visual Data," Southwest Regional Conference for Astronomy and Astrophysics (Editors, Preston F. Gott and Paul S. Riherd). 9, 67-76.

\section{Discussion}

Harry Shipman: Physicists also face the problem of student learning styles and misconceptions that greatly interfere with the learning process and that are hard to deal with through simple lecturing. One solution they use is to use a faculty member in small discussion or problem sessions, either before or at the same time as the faculty member teaches a large lecture. When you teach in a large lecture - talking at rather than with the students - it's easy to remain blissfully ignorant of student misconceptions. Your paper, and the results of Project STAR, suggests that astronomy teachers, even if they have to lecture to hundreds of students, should make efforts to meet regularly with them in smaller groups. 